\title{
Measurement of Neutron Polarization and Transmission for the Neutron EDM at SNS Experiment
}

\section{S. Kavish Imam*i}

The University of Tennessee, Knoxville, TN USA

E-mail: simam@vols.utk.edu

\begin{abstract}
The existence and size of a neutron electric dipole moment (nEDM) remain important questions in particle and cosmological physics. The nEDM@SNS experiment proposes to improve the sensitivity on the nEDM by using polarized ultra-cold neutrons (UCN) and polarized ${ }^{3} \mathrm{He}$ in a bath of superfluid ${ }^{4} \mathrm{He}$. The experiment will employ a cryogenic magnet to provide the required magnetic field environment to achieve the proposed sensitivity. This proceedings will describe a setup to measure the neutron polarization and transmission losses resulting from passage through the magnetic shielding and cryostat windows.
\end{abstract}

The 18th International Workshop on Polarized Sources, Targets, and Polarimetry, PSTP2019 23-27 September, 2019

Knoxville, Tennessee

\footnotetext{
* Speaker.

${ }^{\dagger}$ nEDM@SNS Collaboration
} 


\section{Introduction}

The Standard Model (SM) is the authoritative theory of elementary particles and their interactions. Precisely testing the SM's properties is one of goals of modern particle physics, due to it's lack of explanation for phenomena such as gravitation, dark matter and neutrino masses. Two of the cornerstones of the SM are the Charge-Parity(CP) and Time-reversal(T) symmetries, which are minimally violated in the current SM. The observed matter-antimatter asymmetry in the universe, one of the phenomena unexplained by the SM, requires T/CP violations beyond those found in the SM. Therefore, experimental searches for T/CP symmetry violations beyond the SM are necessary.

The SM predicts an electric dipole moment of the neutron (nEDM), a T/CP symmetry violating quantity, to be $\sim 10^{-32} \mathrm{e} \cdot \mathrm{cm}$. Therefore, a measurement of nEDM would hint at new physics beyond the SM[1]. Experiments with ultra-cold neutron (UCN) have improved the level of precision such that we can constrain theories beyond the SM that predict a large enough T/CP violation. The current experimental limit on the nEDM is $d_{n}<1.8 \times 10^{-26} \mathrm{e} \cdot \mathrm{cm}$ [2]. The next generation of neutron electric dipole moment searches, which plan to achieve sensitivities of $10^{-27} \mathrm{e} \cdot \mathrm{cm}$ to $10^{-28}$ $\mathrm{e} \cdot \mathrm{cm}$, are an important class of experiments aiming to search for new physics in the T-violating sector. Figure 1 shows that nEDM experiments represent one of the most sensitive measurements, which have and will be used to guide theoretical extensions to the SM.

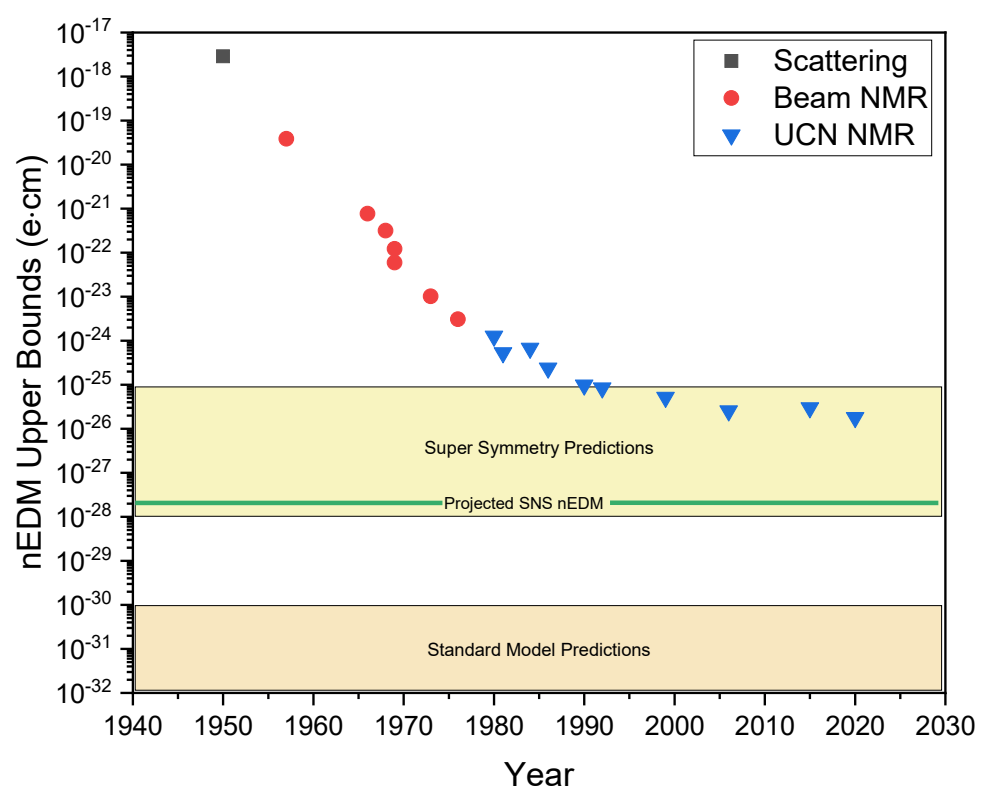

Figure 1: A historical perspective on the observed nEDM upper limits with the date of experiment[4].The latest upper limits measured in 2015[3] and 2020[2] are also shown. The horizontal line indicates the projected goal of nEDM@SNS experiment. The colored regions show the nEDM predicted values from the standard model [1] and supersymmetric models[1].

The primary method for determining the nEDM, $d_{n}$, is to measure the Larmor precession frequency of neutrons with spins aligned in the plane perpendicular to a static magnetic field, $B_{0}$, 
with application of a static electric field, $E_{0}$, parallel/antiparallel to $B_{0}$ :

$$
\omega_{n}=\frac{-2\left(\mu_{n} B_{0} \pm d_{n} E_{0}\right)}{\hbar}
$$

The statistical sensitivity of the nEDM experiment depends on the applied electric field $E_{0}$, the number of neutrons $\mathrm{N}$ and the precession measurement time $\tau$ :

$$
\sigma_{d_{n}} \simeq \frac{1}{E_{0} \tau \sqrt{N}}
$$

To reach a sensitivity of $10^{-28} \mathrm{e} \cdot \mathrm{cm}$, a novel experimental scheme was proposed in [5]. In this setup, polarized ${ }^{3} \mathrm{He}$ and superfluid ${ }^{4} \mathrm{He}$ are used to allow a nEDM measurement to be performed on a high density of polarized UCNs.

\section{Neutron EDM at Spallation Neutron Source}

The nEDM@SNS experiment will be housed at the Spallation Neutron Source(SNS) [8], the most intense pulsed neutron source in the world, to perform an nEDM search using a solution of polarized ${ }^{3} \mathrm{He},\left(X_{3} \sim 10^{-10}\right)$ and $\mathrm{UCN}(\sim 170 U C N / c c)$ in superfluid ${ }^{4} \mathrm{He}$. The experiment will produce UCN via super-thermal scattering of incoming polarized cold neutrons $(\lambda \sim 8.9 \AA)$ off of phonons in superfluid ${ }^{4} \mathrm{He}[6]$. UCN's can be confined to a storage volume made of materials with a Fermi potential less than $165 \mathrm{neV}$. With sufficiently low temperatures and appropriate materials for storage cell walls, UCN can be stored in ${ }^{4} \mathrm{He}$ for long periods of time. This, coupled with the fact that superfluid He has large breakdown voltage and hence allows for application of large electric fields, maximizes $E_{0}, \tau$ and $N$ in eq. 1.2.

${ }^{3} \mathrm{He}$ has a spin-dependent neutron absorption cross section, 5300 barns at $1.8 \AA$ for the singlet state, increasing as $1 / v$, where $v$ is the velocity of neutrons, and nearly zero for the triplet state. The energy released from $n-{ }^{3} \mathrm{He}$ capture decay products is transferred to the liquid helium and produces ultraviolet scintillation light. Because of the spin dependence of the neutron- ${ }^{3} \mathrm{He}$ absorption cross section, the scintillation rate depends on the polarization between $\mathrm{UCN}$ and ${ }^{3} \mathrm{He}$ magnetic moments.

In natural units, the magnetic moment of neutron is 14 orders of magnitude bigger then the proposed nEDM limit. Changes in Larmor frequency due to the magnetic interaction from magnetic field fluctuations will decrease the sensitivity of nEDM. Therefore, the nEDM@SNS sensitivity goal necessitates meticulous control of magnetic field fluctuations. Even under such control, magnetic field gradients are expected but can be taken into account using magnetometry. Since the polarized ${ }^{3} \mathrm{He}$ will be exposed to the same magnetic field as the UCN, and because the Schiff moment of ${ }^{3} \mathrm{He}$ greatly suppresses its EDM, ${ }^{3} \mathrm{He}$ will be utilized as a co-magnetometer.

The nEDM@SNS experiment will employ a multi-pronged strategy to shield against external fields: external field cancellation coils, room-temperature shielding and superconducting shielding [7]. As shown in figure 2, the nEDM magnet cryostat magnet, which surrounds the measurement cells, is made of several concentric layers. The components within the cryogenic magnet package work together to provide shielding and generate magnetic fields that satisfy strict uniformity requirements. Since the nEDM@SNS requires the use of polarized neutrons, therefore, both polarization and transport efficiency of the neutron beam through the cryostat and magnet package windows should be maximized to optimize sensitivity. 


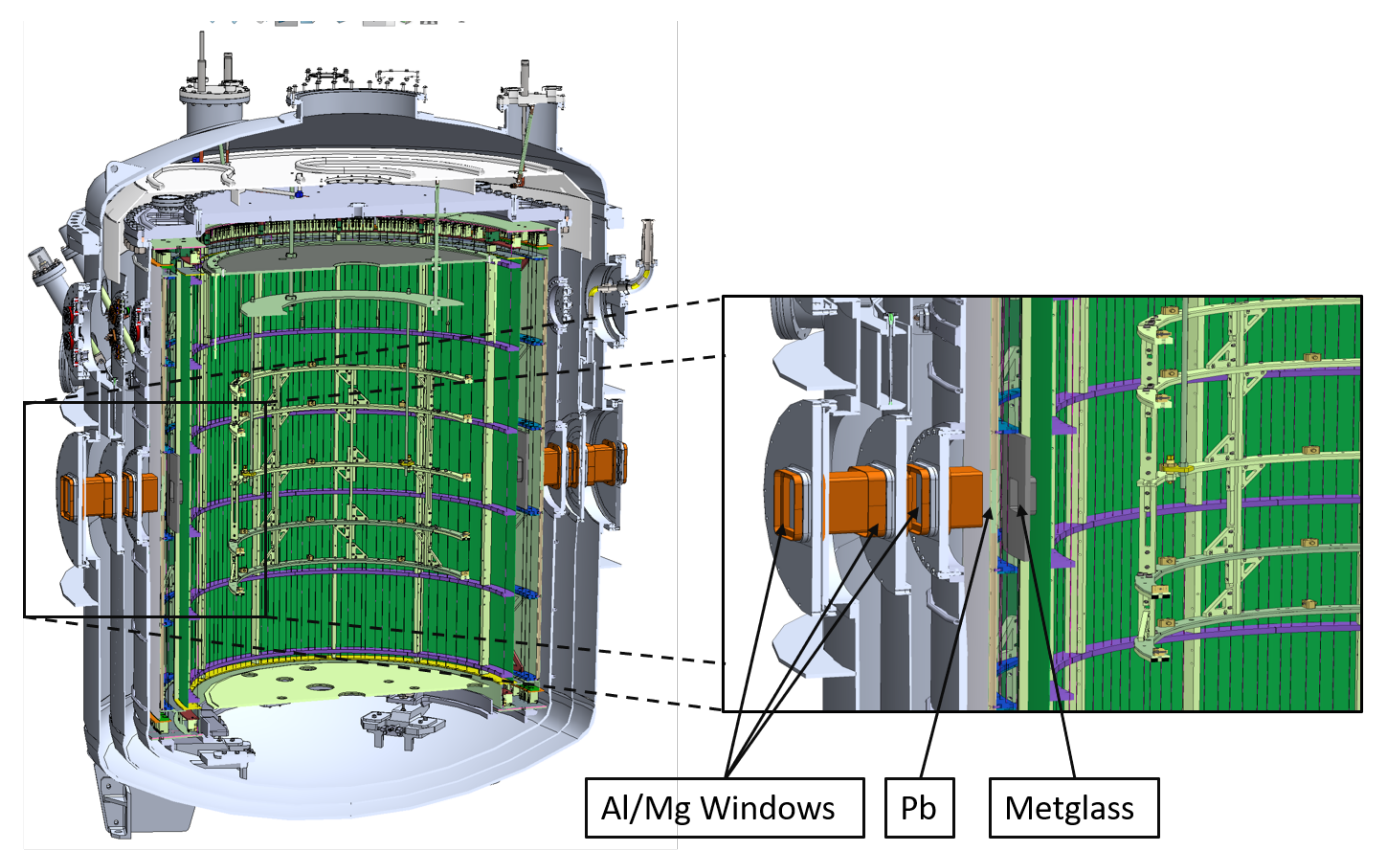

Figure 2: A schematic overview of the nEDM@SNS cryostat magnet package. The enlarged section shows the beam windows the incoming polarized neutrons will pass through.

\section{Neutron Polarization and Transmission Measurements}

Prior to the actual experiment, neutron polarization and transmission measurements will be performed at the SNS Beamline 13A[8]. The neutron beam will traverse the nEDM magnet system via beam windows made of thin metal on the room-temperature vacuum shell and cryogenic thermal shielding, then superconducting $\mathrm{Pb}$, used for improving the magnetic uniformity and for shielding against external AC magnetic fields, then low-cobalt Metglas as a ferromagnetic flux return, then two Si windows serving as the entrance to the central detector volume and a deuterated poly-methyl methacrylate (dPMMA) window of the measurement cell[7]. The objective is to determine the neutron polarization loss and transmission of each of the windows, especially Metglas and $\mathrm{Pb}$, in a similar magnetic field environment of the actual $\mathrm{nEDM}$ experiment. The concern is that the presence of microscopic magnetic domains in the windows and the fact that they can act as current sheets causing a diabatic transition in magnetic field, can lead to small depolarization of neutrons.

\subsection{Neutron Source}

SNS Beamline 13A will be utilized to obtain monochromatic neutrons. It utilizes two Alkaliintercalated graphite monochromators to select $8.9 \AA ̊$ neutrons[8]. Beamline $13 \mathrm{~A}$ continues downstream after the monochromators with an $8 \mathrm{~m}$ expanding ballistic guide to allow for long distance neutron transport[8]. The beamline opens up in the beamline 13 cave wall with a rectangular cross section of $20 \mathrm{~cm} \times 30 \mathrm{~cm}[8]$.

For the PT measurement,a neutron flight tube will be constructed to extend the flight path of the monochromatic neutrons from the existing end of the guide to the nEDM@SNS cryostat magnet 


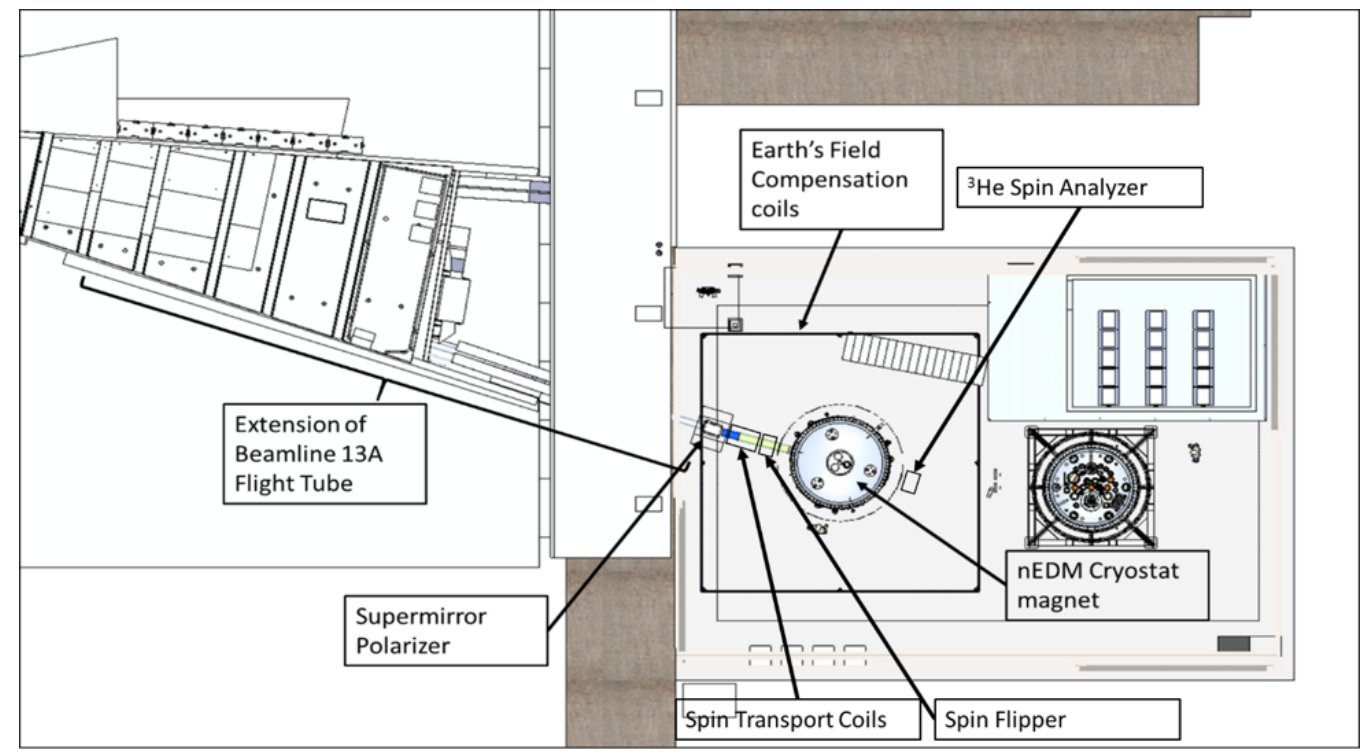

Figure 3: Overview of proposed neutron polarization and transmission measurement for nEDM@SNS experiment at Beamline 13A.

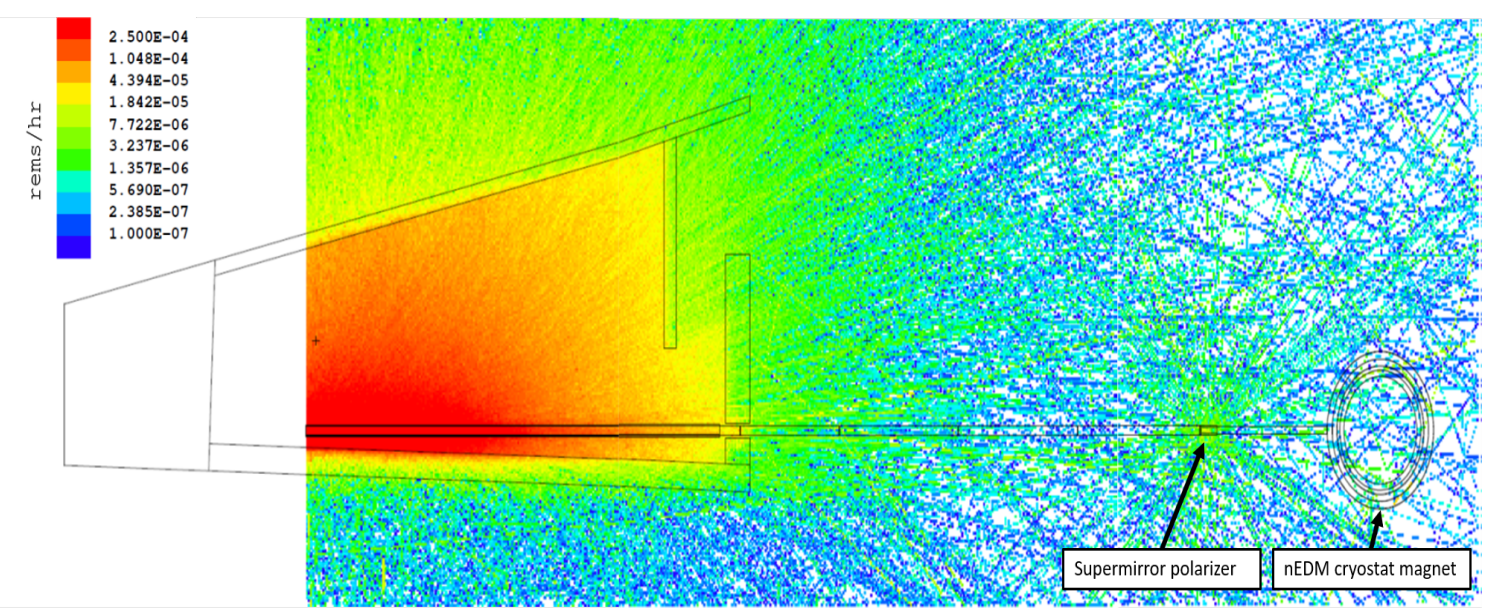

Figure 4: Gamma-rays dose rate from the monochromatic beamline 13A inside and outside the beamline 13 cave walls. The units of dose rate are in rems/hr. In addition to the beamline cave walls, the nEDM@SNS cryostat magnet and supermirror polarizer were also modeled.

as shown in figure 3. The flight tube consists of two regions: i) The region inside the beamline 13 cavewalls will be an aluminum pipe lined up with Mirrobor shielding material. The shielding material has high concentration of ${ }^{10} \mathrm{~B}$ for absorption of diverging neutrons from the beam guide. ii) The flight tube outside the cavewall is made up of an aluminum pipe with $5 \mathrm{LiCO}_{3}$ collimators to reduce the beam size to $5 \mathrm{~cm}$ in radius as well as prevent the diverging beam from creating gamma-ray radiation.

Before the experiment takes place, radiation and dosimetry simulations need to be performed to identify possible personnel safety and radiation shielding requirements. MCNP6 has been utilized to perform these calculations. Figure 4 shows that the dose rate is less then $0.25 \mathrm{mrem} / \mathrm{hr}$, the 
SNS unposted area limit, outside the cave walls, eliminating the need of extra shielding.

\section{2 ${ }^{3}$ He Polarimetry}

A ${ }^{3} \mathrm{He}$ polarization analyzer, which utilizes the spin dependent neutron capture on ${ }^{3} \mathrm{He}$, will be used to measure the polarization of neutrons traversing through the cryostat magnet windows. This can be done by measuring the transmission of (i) polarized neutrons through an unpolarized ${ }^{3} \mathrm{He}$ analyzer, (ii) polarized neutrons through a polarized ${ }^{3} \mathrm{He}$ analyzer, and (iii) spin-flipped neutrons through a polarized ${ }^{3} \mathrm{He}$ analyzer, and (iv) the efficiency of the spin flipper [9]. If we define the ratio of (ii) with (i) as $R$, the ratio of (iii) with (i) as $R_{s f}$ and (iv) as $\varepsilon_{s f}$, eq. 3.1 can be used to determine the polarization of neutron beam, $P_{n}$. $\varepsilon_{s f}$ can be determined by measuring (i), (ii) and transmission of polarized neutrons and spin flipped neutrons through a spin reversed ${ }^{3} \mathrm{He}$ analyzer. The polarization of the ${ }^{3} \mathrm{He}$ cell can be reversed via adiabatic fast passage (AFP).

$$
P_{n}=\frac{R-R_{s f}}{\sqrt{\left[\left(2 \varepsilon_{s f}-1\right) R+R_{s f}\right]^{2}-4 \varepsilon_{s f}^{2}}}
$$

\subsubsection{Neutron Polarizer}

This measurement will utilize a $40 \mathrm{~cm}$ long and with a cross $120 \mathrm{~cm}^{2}$ supermirror polarizer, composed of $450.3 \mathrm{~mm}$ thick panes of borofloat glass with Fe/Si coating of $\mathrm{m}=3$ [10], to polarize the monochromatic neutron beam. The panes have a $9.6 \mathrm{~cm}$ radius of curvature [10] to ensure the incoming neutrons have a glancing angle of incidence. Fe is used for the ferromagnetic layers, and $\mathrm{Si}$ is used for the transparent layers because it has a very small neutron scattering cross section. The field inside the supermirror polarizer is about $350 \mathrm{G}$ which saturates the magnetization of the Fe layers. The magnetization of the Fe determines the magnetic scattering length of the magnetic layers of the supermirror polarizer[10]. The neutrons with angle of incidence greater than the critical angle refract into the borofloat substrate and get absorbed by the natural abundance of ${ }^{10} \mathrm{~B}$.

\subsubsection{Spin Flipper}

We measure the transmission ratio of spin flipped neutrons through the analyzer cell, $R_{s f}$, with a neutron spin flipper. A Mezei coil is well suited to this due to the monochromatic nature of the beam. A Mezei coil flipper works on the principle of diabatic transition of the neutron polarization in a strong DC magnetic field perpendicular to the guide field[11]. A rectangular solenoid of fixed thickness provides the desired magnetic field to induce a torque on the magnetic moment of the polarized monochromatic (i.e. fixed velocity) neutron for a $\pi$ radians flip.

\subsection{3 ${ }^{3}$ He analyzer}

${ }^{3} \mathrm{He}$ has a very strong spin dependent neutron absorption cross section, 5300 barns at $1.8 \AA$ for singlet state and nearly zero for triplet state, to give the reaction ${ }^{3} \mathrm{He}(\mathrm{n}, \mathrm{p}) \mathrm{t}$. Glass cells filled with polarized ${ }^{3} \mathrm{He}$ are commonly used as spin analyzers for polarized neutrons[12]. The ${ }^{3} \mathrm{He}$ nuclei are typically polarized using spin exchange optical pumping, where circularly polarized light is used to optically pump electrons from alkali atoms into higher angular momentum states. Through weak hyperfine interactions, the high angular momentum state electrons transfer their spin to ${ }^{3} \mathrm{He}$ 
nuclei. These cells can be constructed to encompass the entire beam area and ${ }^{3} \mathrm{He}$ polarization can be maintained in a magnetic field for several hours or continuously under in situ polarization.

\section{Summary}

The nEDM@SNS experiment aims to search for the neutron EDM to a sensitivity of $10^{-28}$ e.cm. This limit will establish constraints on theories that propose explanations for matter-antimatter asymmetry. The nEDM@SNS experiment plans to achieve the above mentioned sensitivity by producing polarized UCN in situ superfluid helium as well as performing live spin analysis of UCN using polarized ${ }^{3} \mathrm{He}$. These techniques require a strict control over the magnetic fields, and the experiment will employ a cryogenic magnet package to achieve this. Simulation of neutron polarization transitions through magnetic materials are difficult therefore, polarization loss has to be measured experimentally. ${ }^{3} \mathrm{He}$ polarimetry is commonly utilized for neutron polarization analysis, therefore, becomes an ideal choice for the polarization and transmission measurements.

The cryogenic magnet package is currently being commissioned at California institute of Technology and is expected to arrive at SNS in 2021. Presently, all polarimetry components are being constructed, engineering design of the flight tube is being performed and radiation shielding review for beamline 13 is in process.

\section{References}

[1] D. Dubbers and M. G. Schmidt, The neutron and its role in cosmology and particle physics Rev. Mod. Phys. 83, (2011) 1111.

[2] C. Abel et al., Measurement of the Permanent Electric Dipole Moment of the Neutron Phys. Rev. Lett. 124, (2020) 081803.

[3] J. M. Pendlebury et al., Revised experimental upper limit on the electric dipole moment of the neutron Phys. Rev. D 92, (2015) 092003

[4] S. K. Lamoreaux and R. Golub Experimental searches for the neutron electric dipole moment J. Phys. G: Nucl. Part. Phys. 36, (2009) 104002.

[5] R. Golub and S. K. Lamoreaux, Neutron electric dipole moment, ultracold neutrons and polarized ${ }^{3} \mathrm{He}$ Phys. Rept. 273, (1994) 1.

[6] R. Golub, J. M. Pendlebury, The interaction of ultra-cold neutrons (UCN) with liquid helium and a superthermal UCN source Phys. Lett. A 62 , (1977) 337.

[7] M. Ahmed et al. A new cryogenic apparatus to search for the neutron electric dipole moment $\mathbf{J}$. Instrum, 14, (2019) P11017.

[8] N. Fomin et al. Fundamental Neutron Physics Beamline at the Spallation Neutron Source at ORNL. Nucl. Instrum. Methods Phys. Res. A 773 (2015) 45.

[9] G. L. Greene, A. K. Thompson, M. S. Dewey, A method for the accurate determination of the polarization of a neutron beam using a polarized ${ }^{3}$ He spin filter. Nucl. Instrum. Methods Phys. Res. A 365 (1995) 177.

[10] S. Balascuta, et al. The implementation of a super mirror polarizer at the SNS fundamental neutron physics beamline. Nucl. Instrum. Methods Phys. Res. A 671 (2012) 137. 
[11] F. Mezei, Neutron spin echo: A new concept in polarized thermal neutron techniques. Z. Physik, 255 (1972) 146.

[12] T. Chupp, et al., Polarized, high density, gaseous ${ }^{3}$ He targets. Phys. Rev. C 36, (1987) 2244. 